\title{
Multi-Messenger Studies of Cosmic-Ray - Acceleration in Galaxy Cluster Accretion Shocks
}




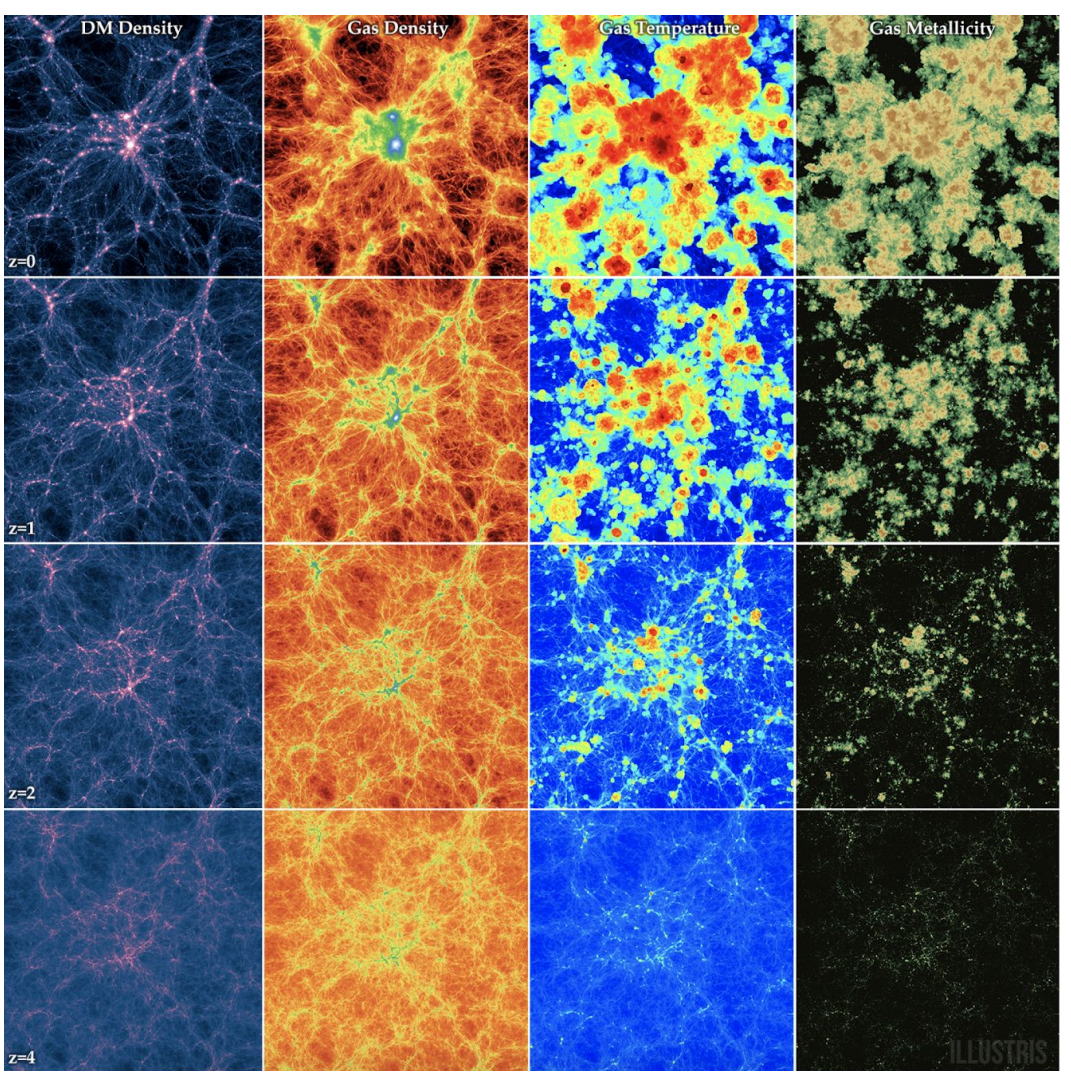

https://www.illustris-project.org
- Galaxy clusters - largest gravitationally bound objects in the Universe.

- We can learn about them from simulation and multiwavelength observations.

- Galaxy formation and evolution, dark matter, gravitational lensing, big bang and the evolution of the cosmos, chemistry .... and ... particle acceleration.

\section{Coma cluster}

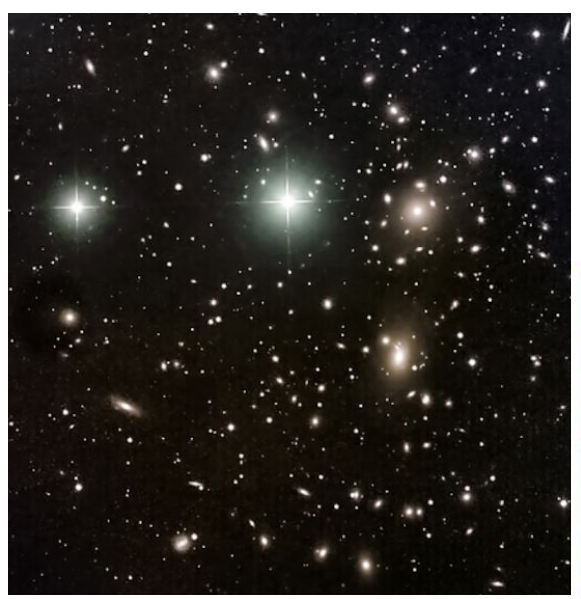

Over 1000 galaxies $z=0.02$ or $103 \mathrm{Mpc}$ (336 Mly)

tSZ X-ray

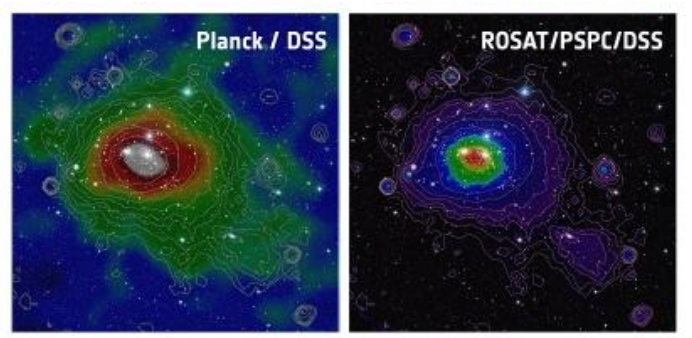


- Diffusive shock acceleration - origin of highest energy particles in the Universe (far above what we can produce in accelerators on Earth).

- Recipe for particle acceleration ingredients: particles to accelerate, magnetic field, shock wave.

- Ingredients are present in different environments and scales - supernova remnants, Solar wind termination shock, ANGs, gamma-ray bursts ... and ... large scale gas accretion.

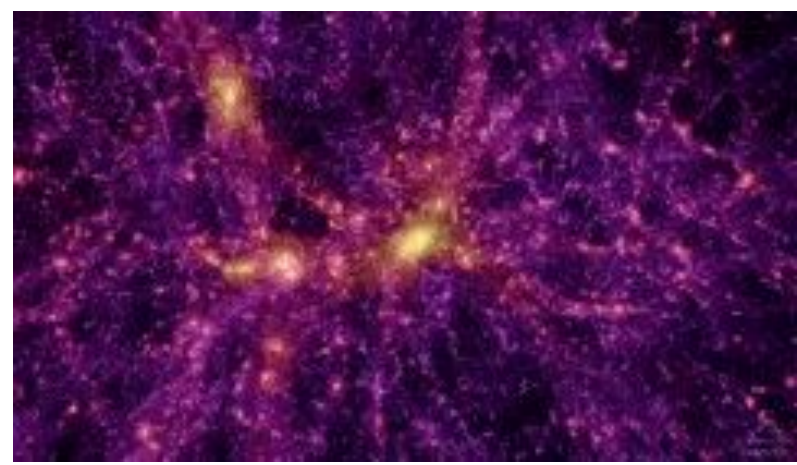

\section{Particle acceleration - Cosmic Rays}

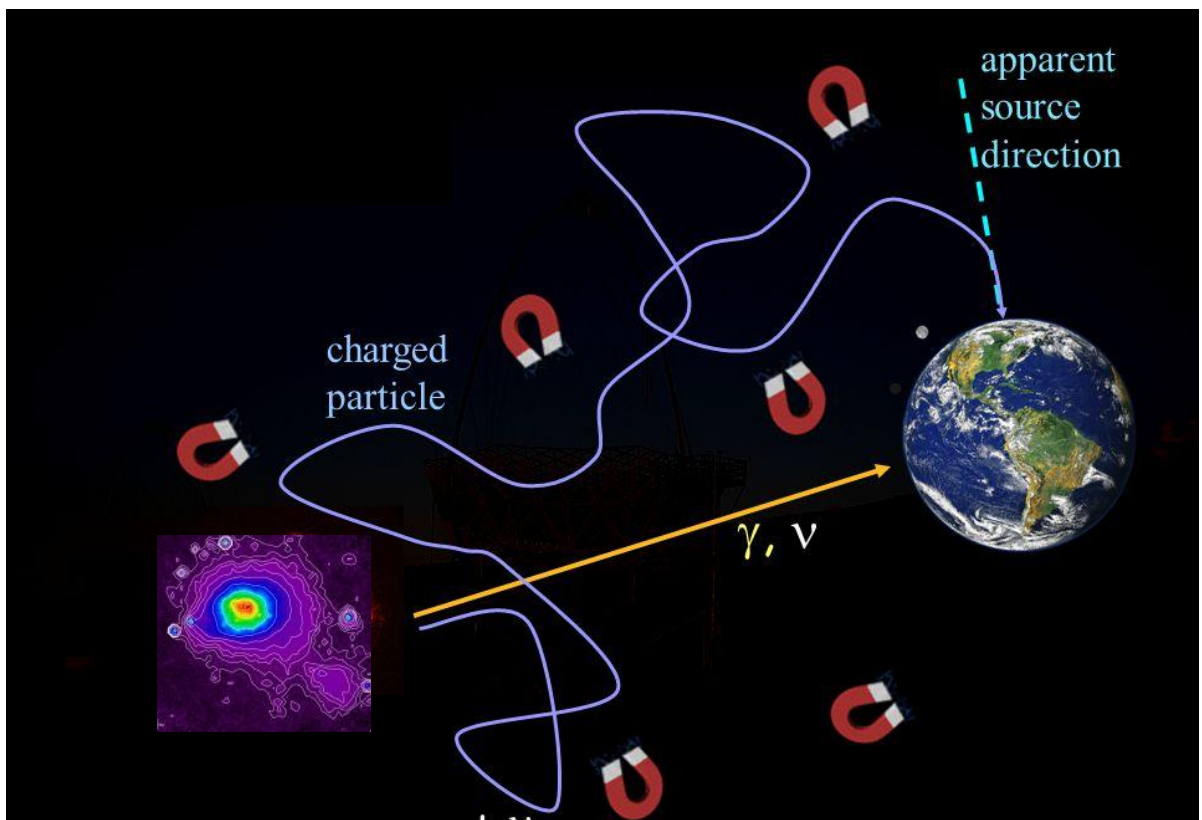

How do we learn about the source using CRs? 


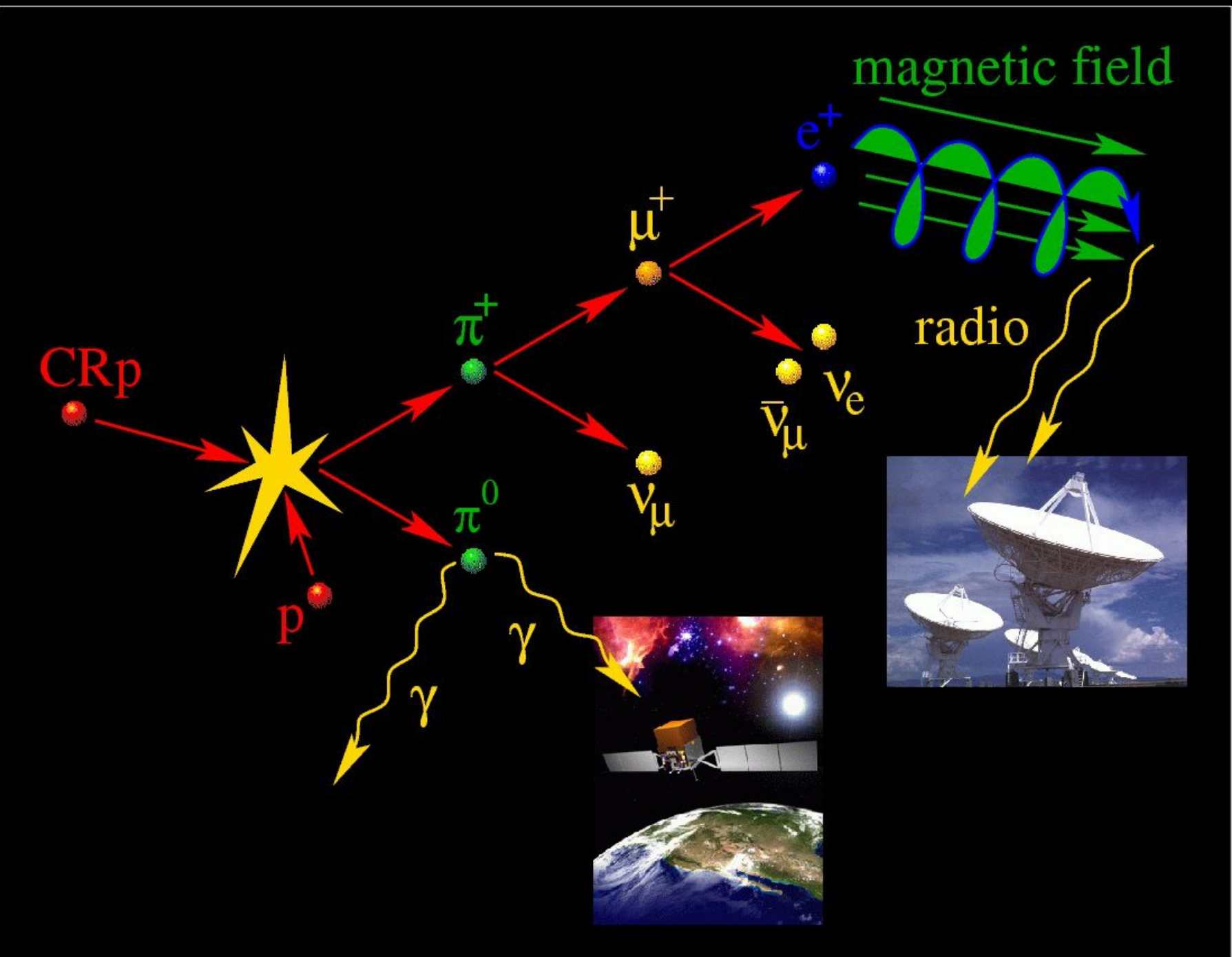




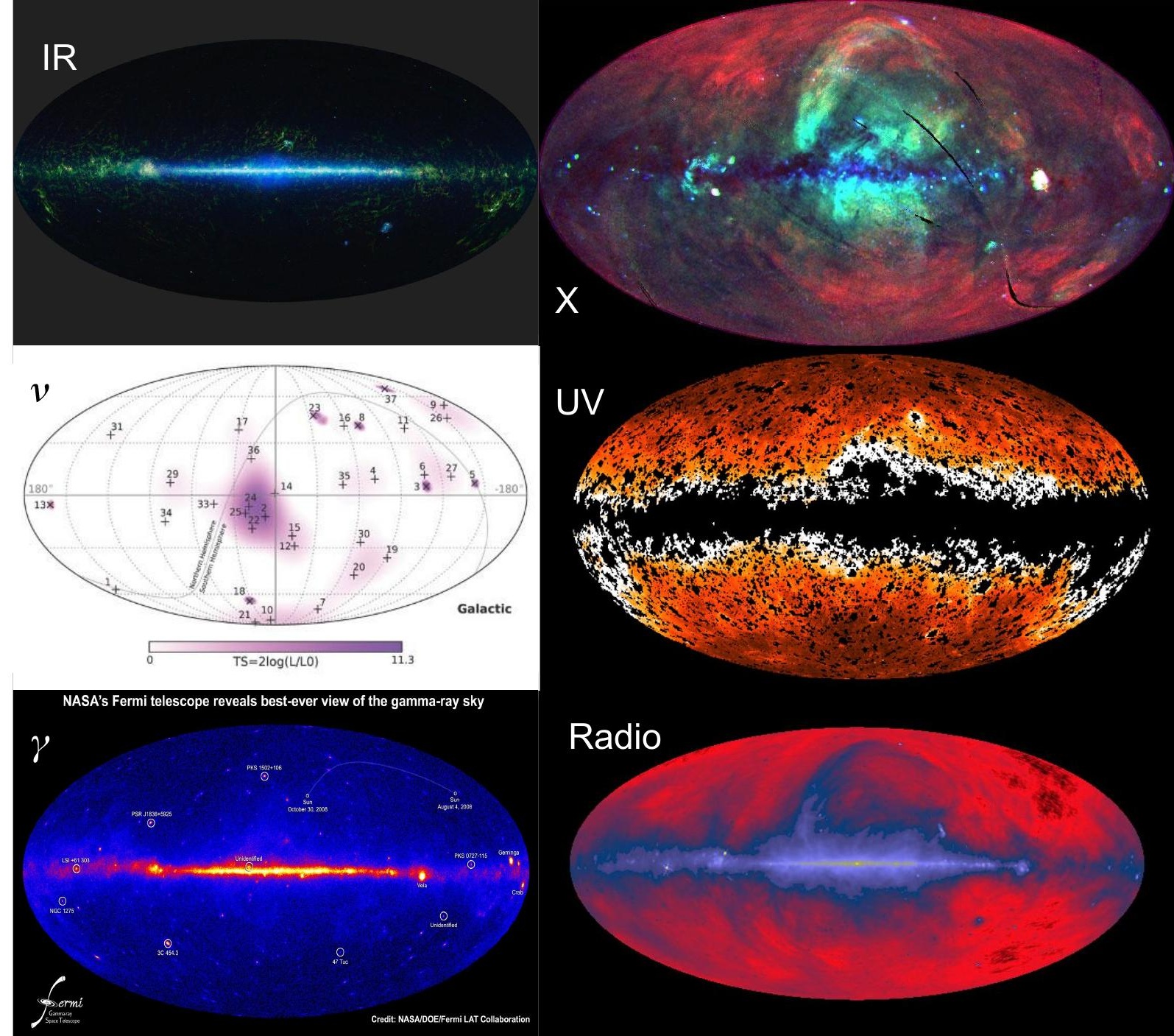




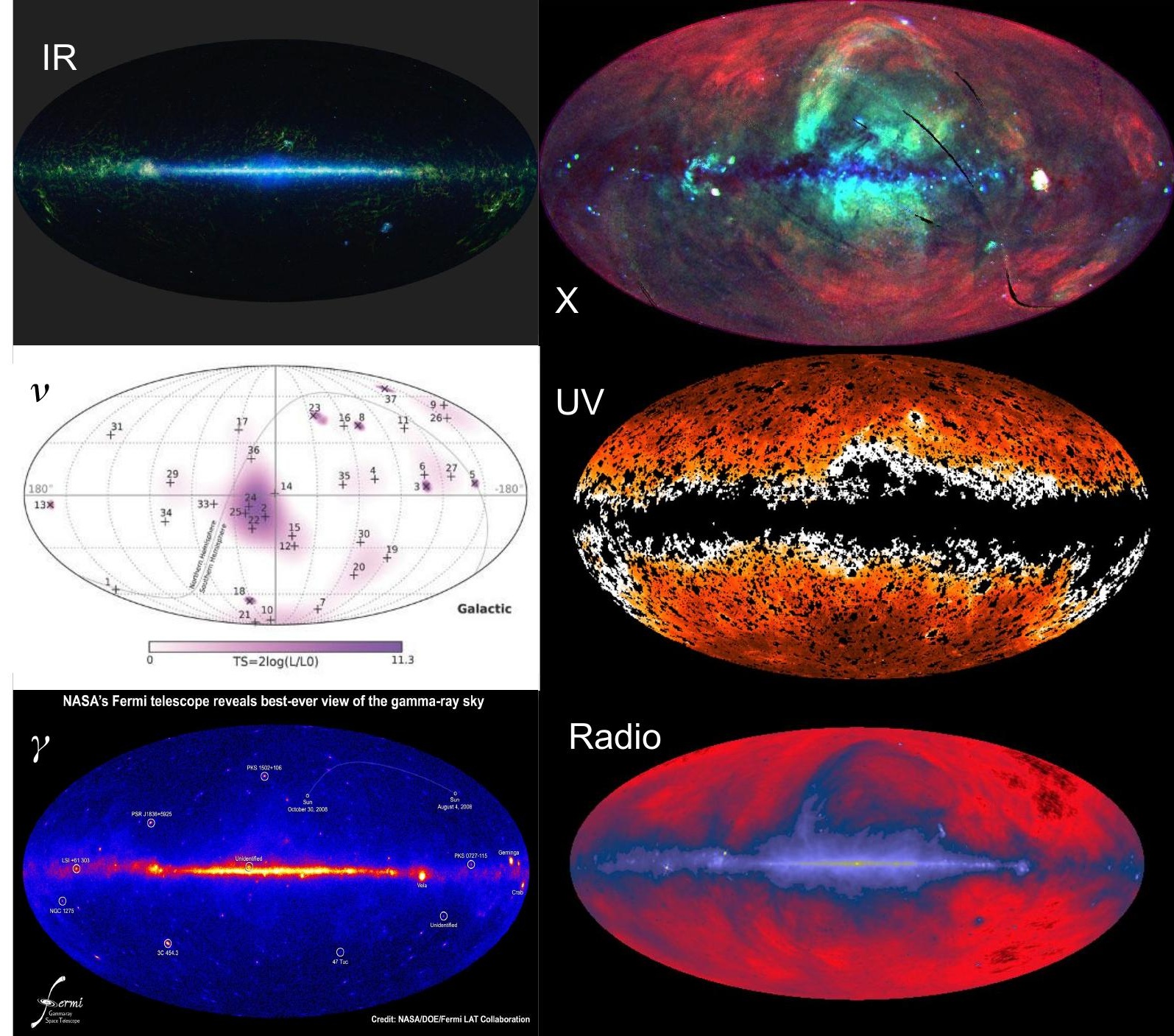


Connecting the dots...

Gamma Rays + Neutrinos

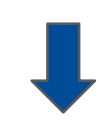

Radio waves $+\mathrm{tSZ}$

Conclusion
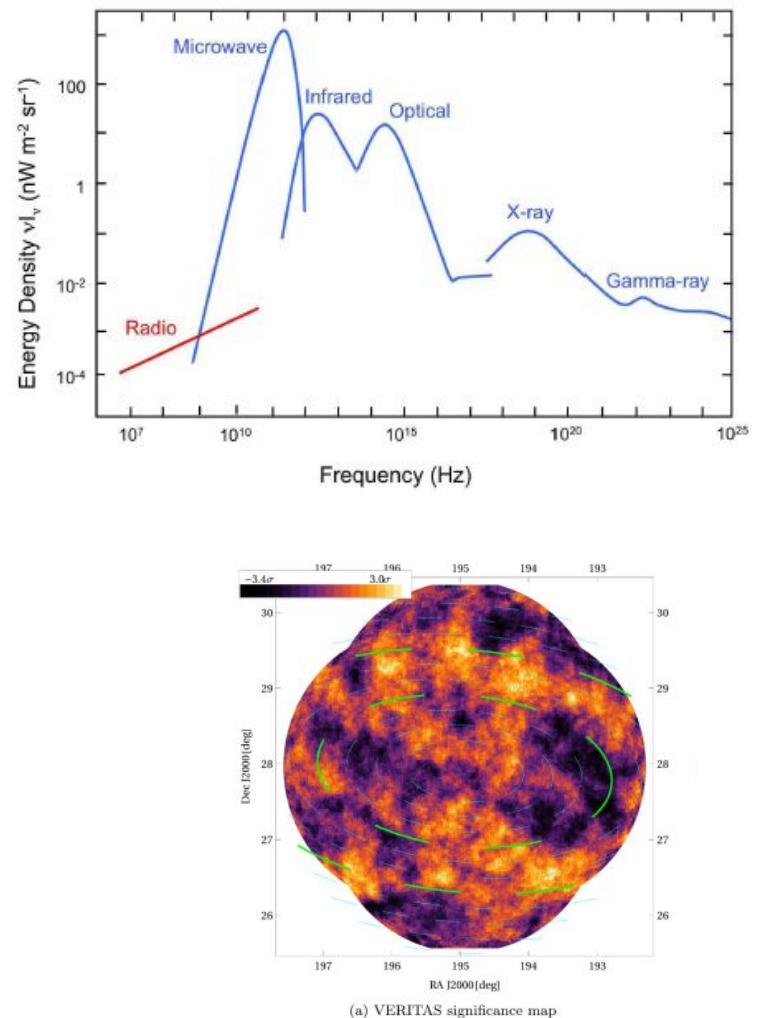

Gamma ring around Coma cluster [Keshet, U. et al. 2017, ApJ, 845, 24]
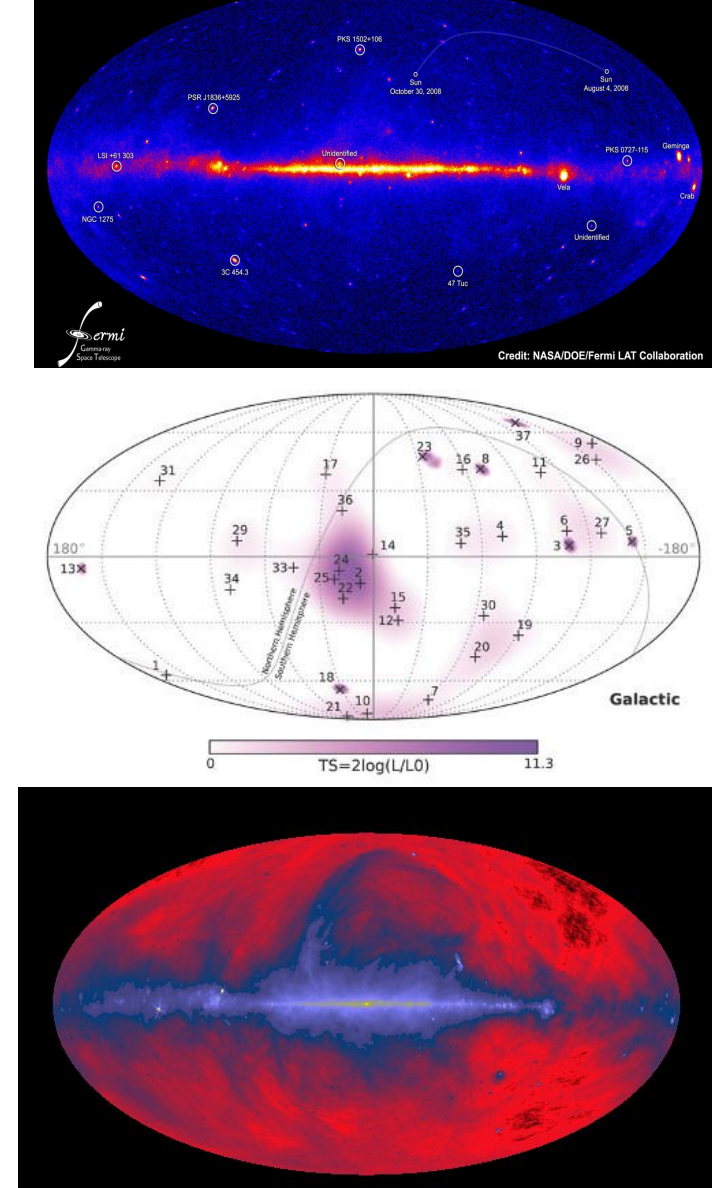


\section{Gamma-Rays and Neutrinos}

$\mathrm{I}_{\gamma}(\mathrm{E}) \propto$ cosmology $\mathbf{x}$ cosmic accretion rate $\mathbf{x}$ gas fraction $\mathbf{x}$ gamma-ray spectrum $\mathbf{x}$ normalisation

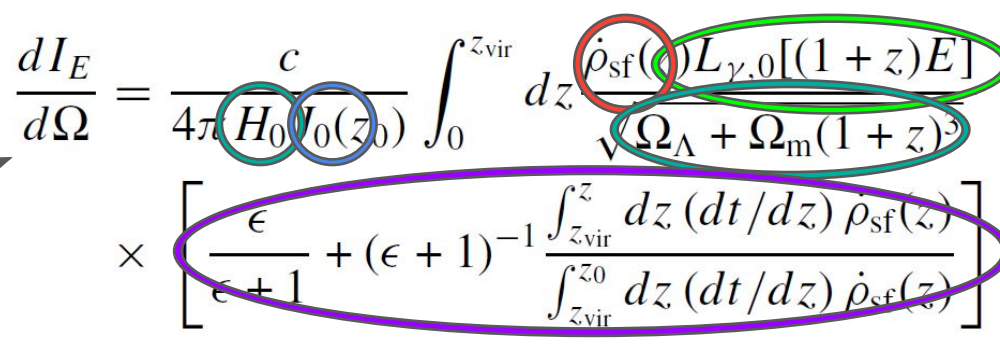

- Differential intensity $\left[\mathrm{cm}^{-2} \mathrm{~s}^{-1} \mathrm{GeV}^{-1} \mathrm{sr}^{-1}\right]$

- Evolution of accretion shocks throughout the history of the Universe - analytical models [Pavidou \& Fields 2006, ApJ, 642, 734]

- Cosmic accretion rate $-\mathrm{J}$ or $\varrho_{\mathrm{sf}}$

- Unresolved galaxy clusters - contribution to isotropic gamma-ray background (Fermi - LAT) + normalization using neutrinos (IceCube).

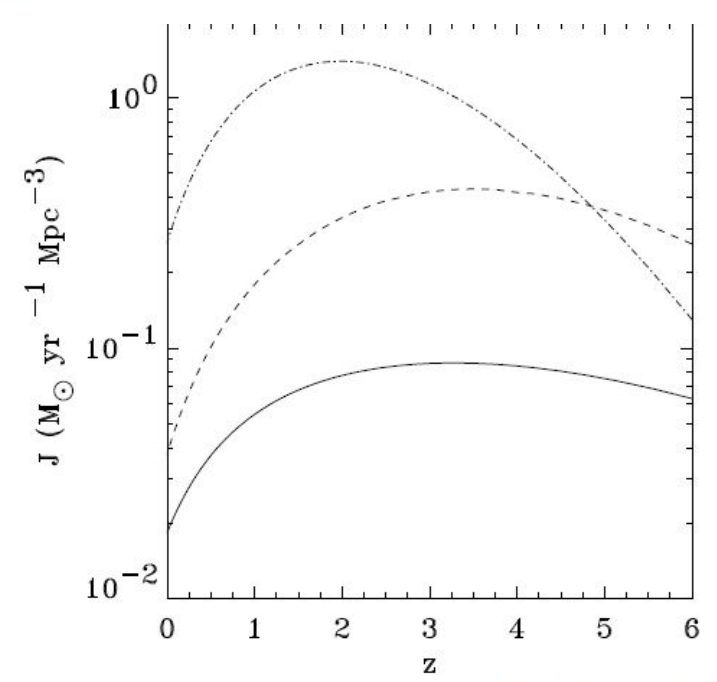




\section{Gamma-Rays and Neutrinos}

- Assumption 1: evolution of cosmic accretion rate directly translates to acceleration of CRs, and the resulting gamma-ray, neutrino, radio waves production etc.

- Assumption 2: gamma-rays are mostly from neutral pions - spectral shape is broken power law

[Pfrommer \& Enlin 2003, A\&A, 407, 73]
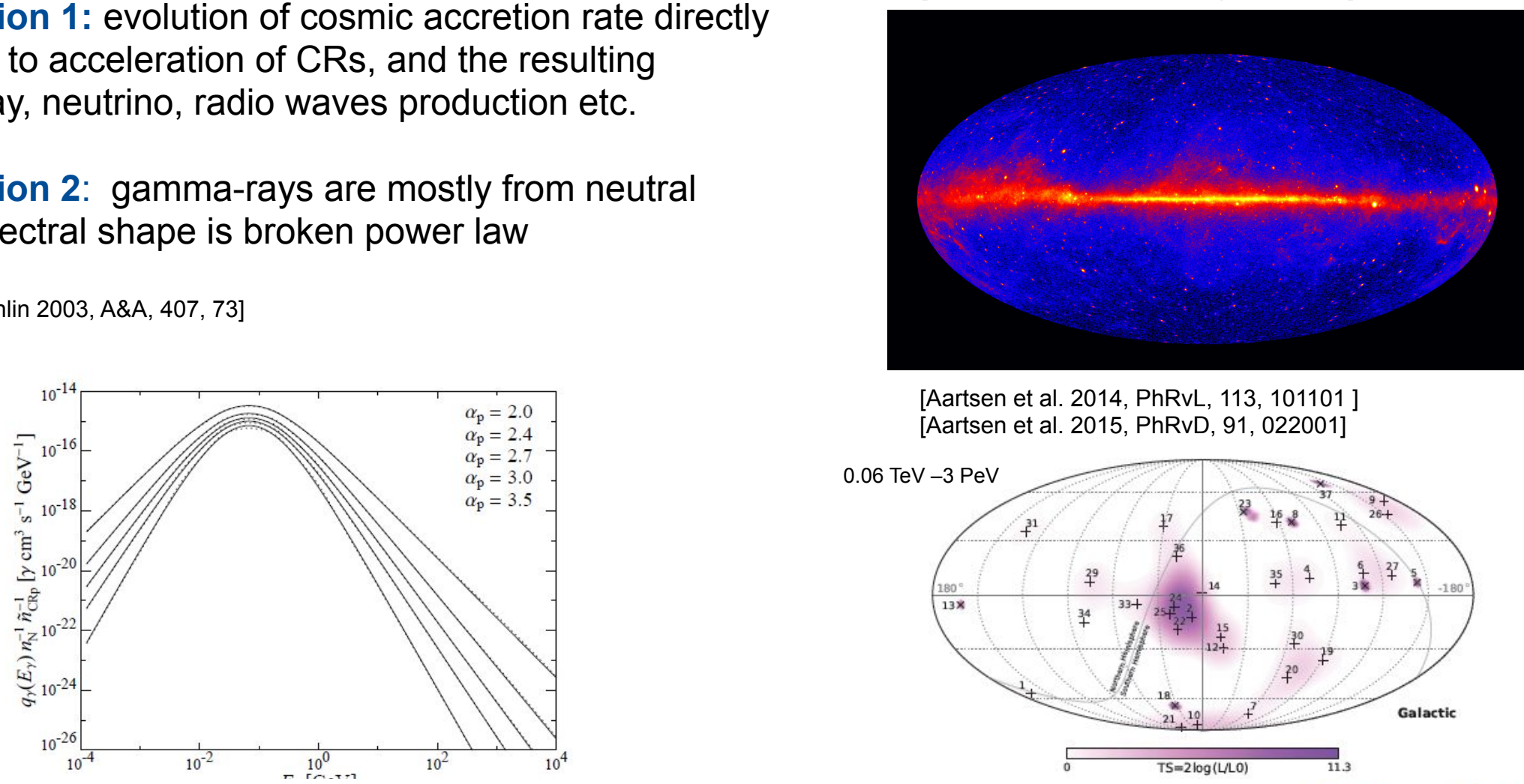

[Aartsen et al. 2014, PhRvL, 113, 101101] [Aartsen et al. 2015, PhRvD, 91, 022001]

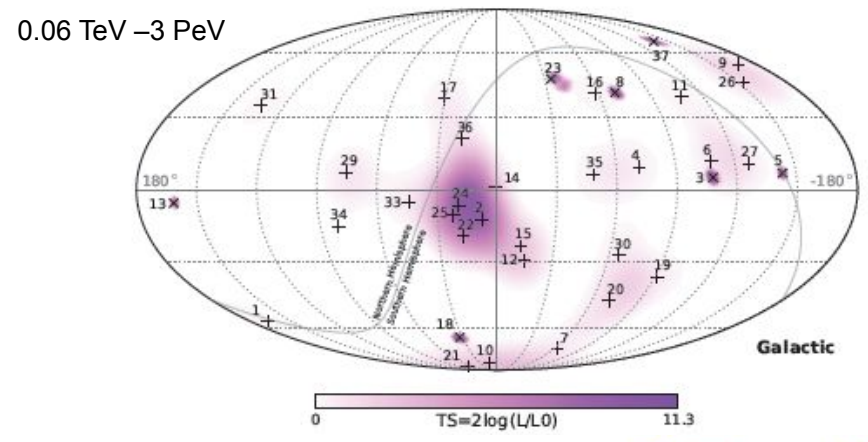




\section{Gamma-Rays and Neutrinos}

- Neutrinos are produced via charged pion decay, while gamma rays have origin in neutral pion decay.

- Neutrino - gamma rays link is simple:

$$
\begin{aligned}
& \frac{\mathrm{d} N_{\gamma}}{\mathrm{d} E_{\gamma}}=\frac{2^{\alpha}}{6} \frac{\mathrm{d} N_{\nu}}{\mathrm{d} E_{\nu}}\left(E_{0}\right) \\
& (\alpha=2): \quad E^{2} \frac{\mathrm{d} N_{\gamma}}{\mathrm{d} E_{\gamma}}(E)=2 E^{2} \frac{\mathrm{d} N_{\nu_{i}}}{\mathrm{~d} E_{\nu_{i}}}(E) \mid E=E_{0}
\end{aligned}
$$

[Ahlers \& Murase 2014, PhRvD, 90, 023010]

[Chang \& Wang 2014, ApJ, 793, 131]

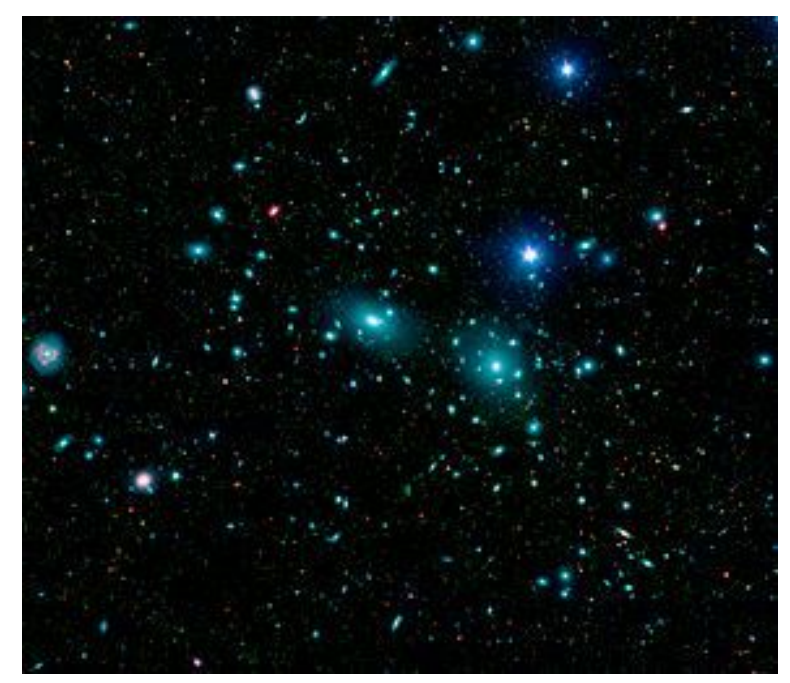




\section{Gamma-Rays and Neutrinos}

- If accretion shocks are predominantly strong neutrinos are much more constraining.

- Accretion shocks can have possible non-negligible contribution to diffuse backgrounds.

- Upper limits to clusters + SF galaxies IGRB contribution $>30-40 \%(100 \mathrm{GeV})$

[Murase, Ahlers \& Lacki 2013, PhRvD, 88, 121301]

- Less than $20 \%$ of the neutrinos could be from clusters?
[Dobardžić \& Prodanović 2014, ApJ, 782, 109]

[Dobardžić \& Prdanović 2015, ApJ, 806, 184]

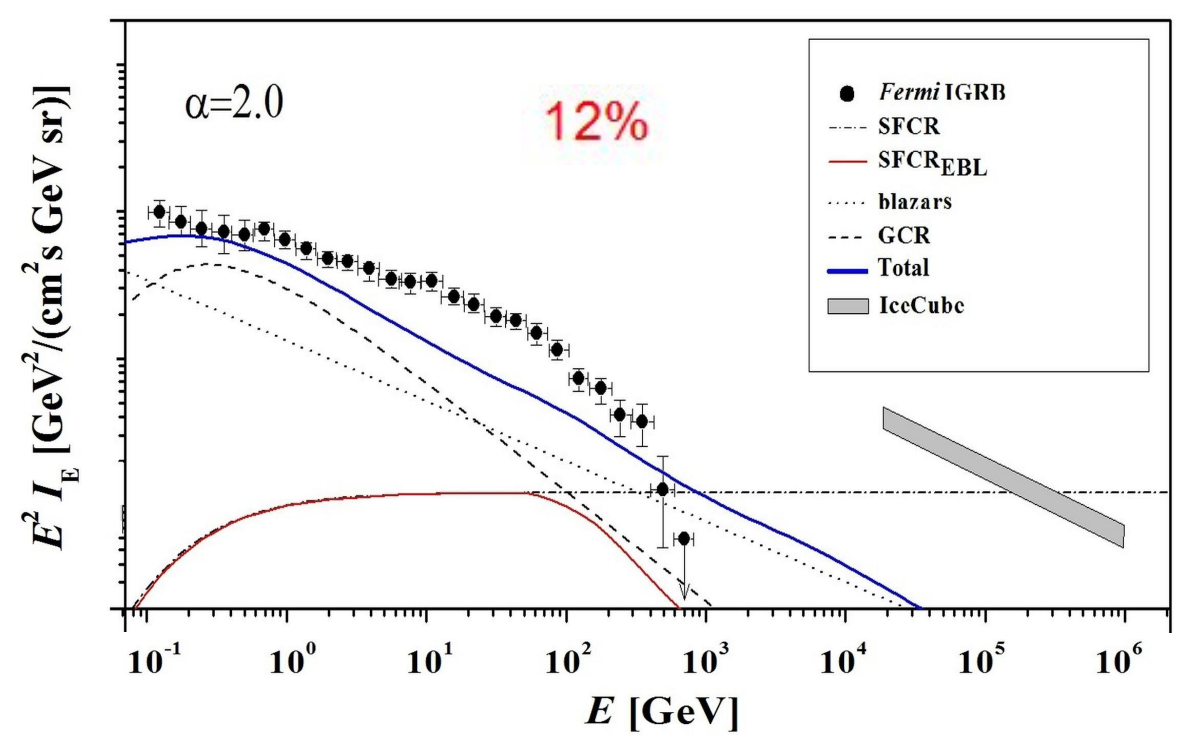




\section{$\underline{\text { Radio and tSZ }}$}

- CR electrons produce synchrotron radiation in radio domain.

- $\quad$ CRB spectrum is a power law with index -2.6 .

- Regular galaxies, big radio galaxies, AGNs, quasars, galaxy clusters, radio supernovae, diffuse sources, dwarf galaxies, low surface brightness sources...

- Managing to explain only $67 \%$ CRB at 1.4 GHz. [Draper et al. 2011]

- We can use the same models as in case of gamma rays to get the contribution of galaxy clusters to the CRB.
Cosmic Radio Background - CRB

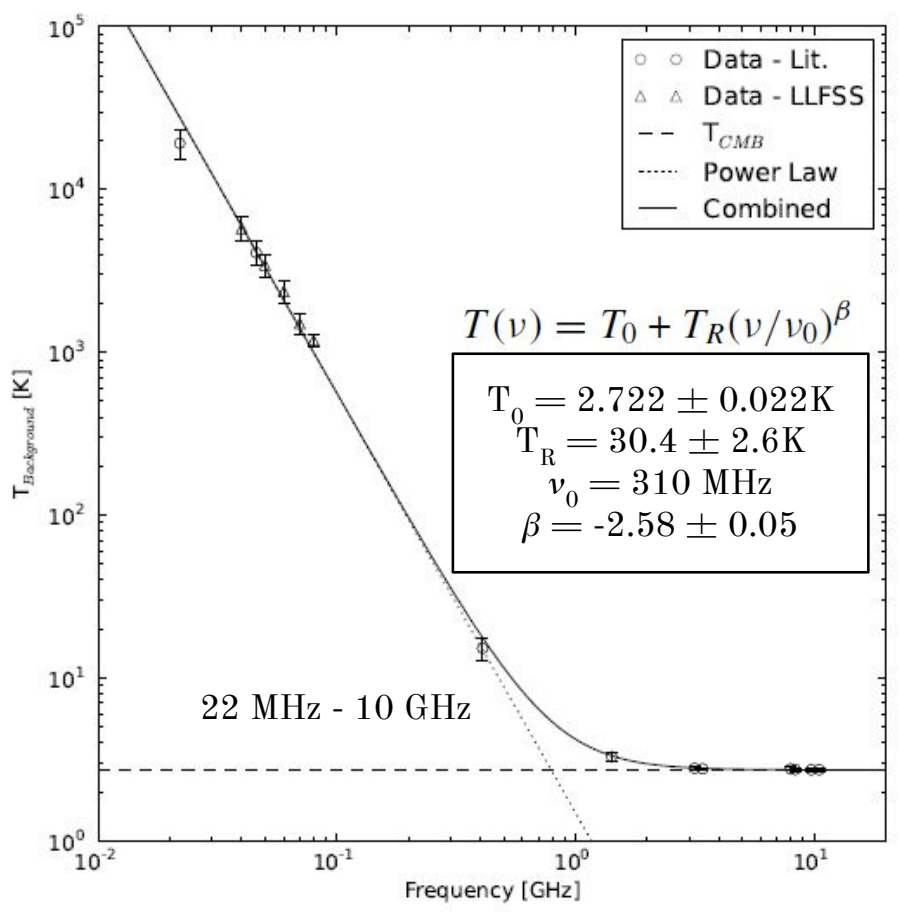

ARCADE 2 measurements Long Wavelength Array [Fixsen, D. J. et al. 2011, ApJ, 734, 5] [Dowell \& Taylor 2018] 


\section{Origin of high-energy CRs in galaxy clusters?}

\section{HADRONIC MODELS}

$\mathrm{p}_{\mathrm{cr}}+\mathrm{p} \rightarrow \pi^{ \pm} \rightarrow \mathrm{e}^{ \pm}+v_{\mathrm{e}} \bar{v}_{\mathrm{e}}+v_{\mu}+\bar{v}_{\mu}$

- $\mathrm{CR}$ accelerated in accretion shocks, AGNs....

- Power law spectrum.

\section{REACCELERATION OF ELECTRONS}

- Electrons that already exist in clusters with 0.1-10 GeV are accelerated above $10 \mathrm{GeV}$ in turbulences during cluster interactions.

- But electrons lose energy fast and acceleration is not efficient. 


\section{Origin of high-energy CRs in galaxy clusters?}

\section{HADRONIC MODELS}

$\mathrm{p}_{\mathrm{cr}}+\mathrm{p} \rightarrow \pi^{ \pm} \rightarrow \mathrm{e}^{ \pm}+v_{\mathrm{e}} \bar{v}_{\mathrm{e}}+v_{\mu} \overline{+} v_{\mu}$

- $\mathrm{CR}$ accelerated in accretion shocks, AGNs....

- Power law spectrum.

\section{REACCELERATION OF ELECTRONS}

- Electrons that already exist in clusters with 0.1-10 GeV are accelerated above $10 \mathrm{GeV}$ in turbulences during cluster interactions.

- But electrons lose energy fast and acceleration is not efficient. 


\section{$\underline{\text { Radio and tSZ }}$}

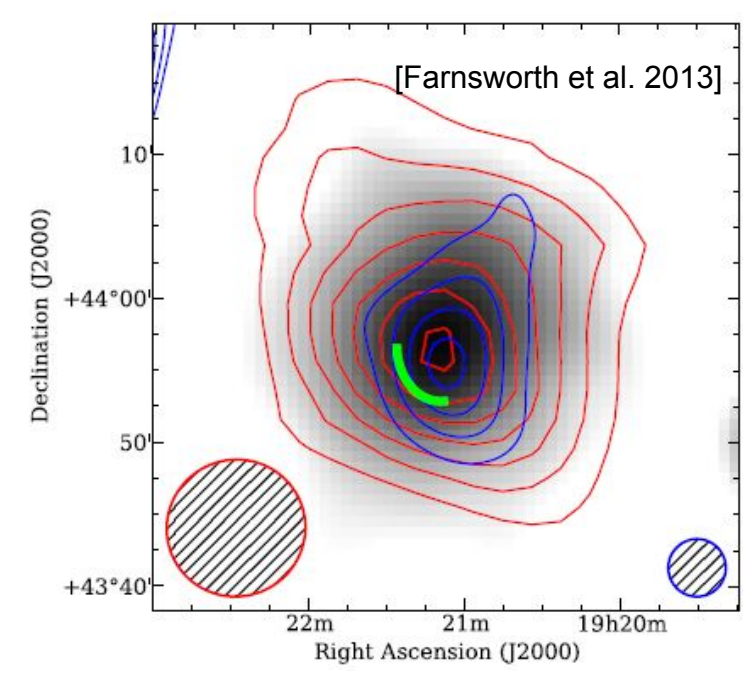

Green Bank Telescope $1.4 \mathrm{GHz}$

VLA $1.4 \mathrm{GHz}$

ROSAT All Sky Survey

XMM-Newton
Radio spectrum from observations

$\mathrm{L}(v)=\mathrm{L}_{1.4}(v / 1.4 \mathrm{GHz})^{-\alpha}$

$0.4-1.4 \mathrm{GHz}$

$\alpha=1.2$

[Farnsworth et al. 2013]

$\alpha=1.8$

[Feretti et al. 1997]

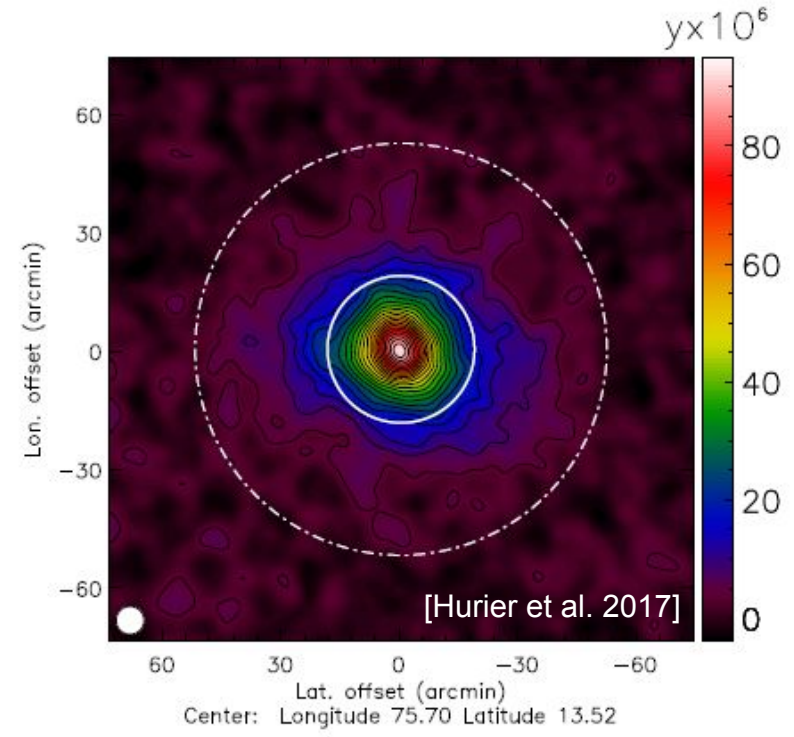

tSZ - location of the virial shock, current accretion rate $R_{v}=(2.93 \pm 0.05) \times R_{500}$ $J_{0}=(1.4 \pm 0.4) \times 10^{5} \mathrm{M}_{\text {sun }} \mathrm{yr}^{-1}$ 


\section{Contribution of unresolved galaxy clusters to CRB}

- A2319 was observed et several frequencies. Different authors derive slightly different power law spectra for A2319.

[Farnsworth, D. et al. 2013, ApJ, 779, 189]

[Feretti, L., Giovannini, G., Böhringer, H. 1997, NewA, 2, 501]

- At $1.4 \mathrm{GHz}$ the possible contribution of galaxy clusters is less than around $1 \%$, and in the 0.02-10 $\mathrm{GHz}$, where CRB is measured, $<1-5 \%$.

- If not all CR electrons located at the outskirts of the cluster are from virial shock than $J_{0}$ would be overestimated, but not for much since then we would overshoot the CRB at lowest frequencies.

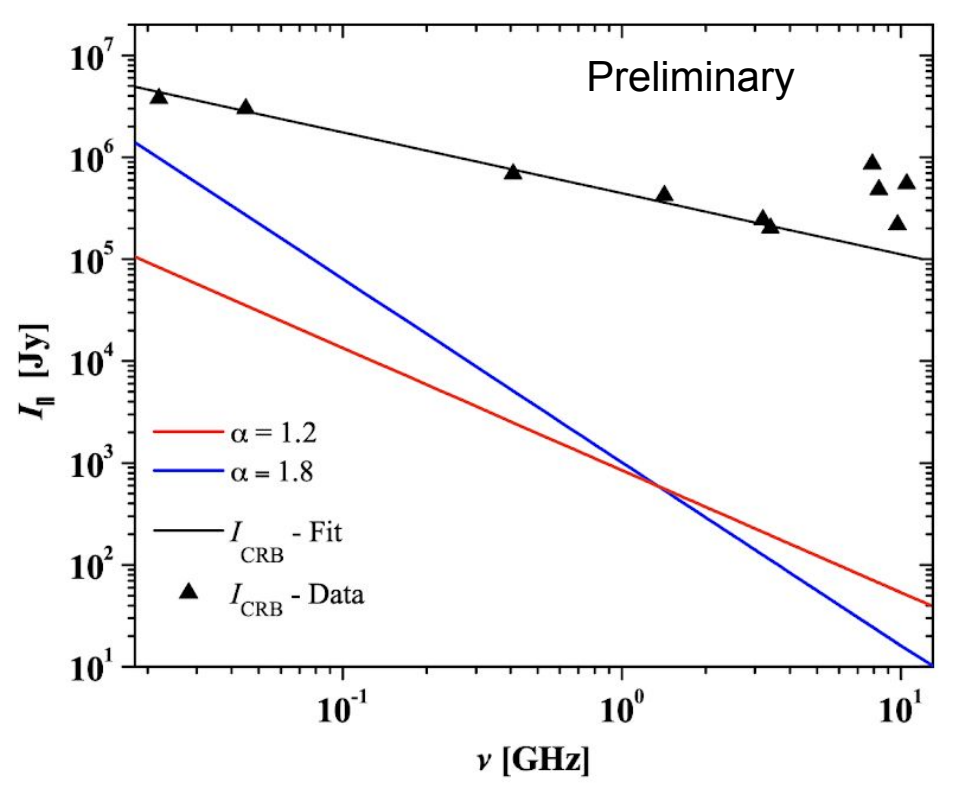




\section{Conclusion}

- Galaxy clusters are probes of large scale particle acceleration.

- Acceleration of particle in accretion shocks is still not well understood.

- Only by leveraging multi-messenger studies we'll be able to better understand processes that lead to $C R$ acceleration, their properties and influence on evolution of astrophysical objects.

- We need new and better observations, and to find more visible galaxy clusters in different observations.

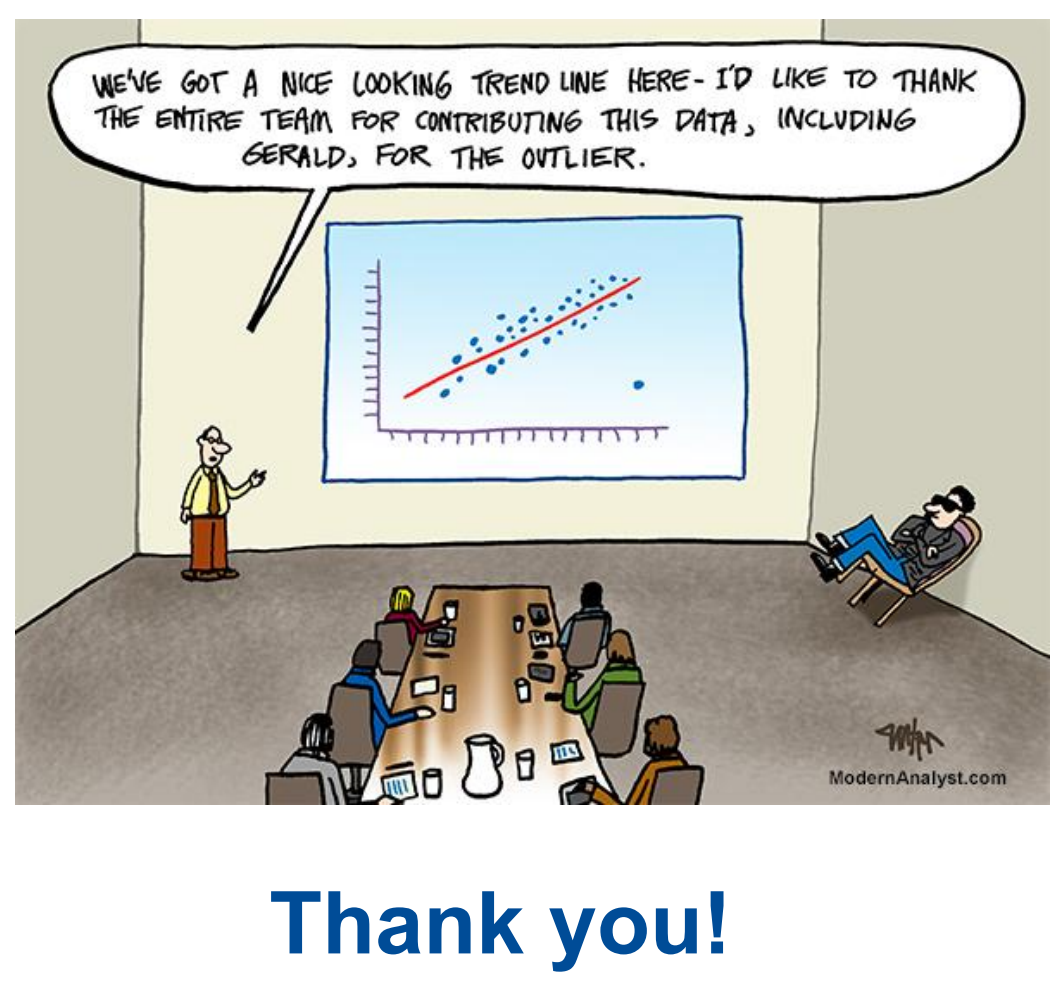

